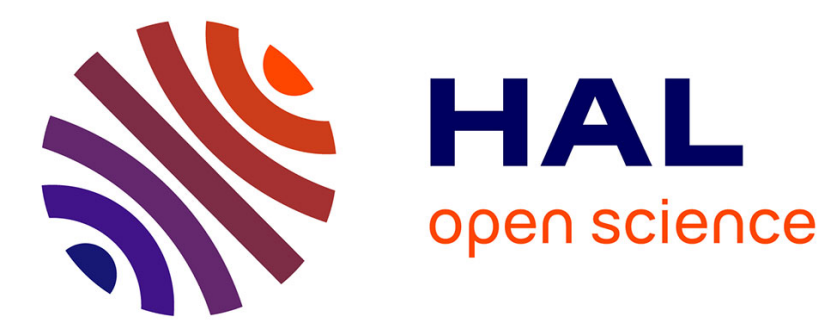

\title{
Une grammaire locale de l'expression des sentiments
} Maurice Gross

\section{To cite this version:}

Maurice Gross. Une grammaire locale de l'expression des sentiments. Langue française, 1995, 105 (1), pp.70-87. hal-00621434

\section{HAL Id: hal-00621434 \\ https://hal.science/hal-00621434}

Submitted on 10 Sep 2011

HAL is a multi-disciplinary open access archive for the deposit and dissemination of scientific research documents, whether they are published or not. The documents may come from teaching and research institutions in France or abroad, or from public or private research centers.
L'archive ouverte pluridisciplinaire HAL, est destinée au dépôt et à la diffusion de documents scientifiques de niveau recherche, publiés ou non, émanant des établissements d'enseignement et de recherche français ou étrangers, des laboratoires publics ou privés. 


\section{Une grammaire locale de l'expression des sentiments}

In: Langue française. $N^{\circ} 105,1995$. pp. 70-87.

Abstract

We present the grammar of a set of sentences that express "feelings". We consider two main types : sentences with two arguments, one is a person and the other is the "feeling" affecting this person. We study all syntactic patterns that correspond to such semantically defined utterances. The second type of sentences is causative with respect to the preceding one, hence a third argument is introduced : the noun phrase that triggers the "feeling". We study again the syntactic patterns available in French to express this situation and the syntactic transformations that relate both types.

Citer ce document / Cite this document :

Gross Maurice. Une grammaire locale de l'expression des sentiments. In: Langue française. №105, 1995. pp. 70-87.

doi : $10.3406 /$ lfr. 1995.5294

http://www.persee.fr/web/revues/home/prescript/article/lfr_0023-8368_1995_num_105_1_5294 
Maurice Gross

Université Paris 7

Laboratoire d'Automatique Documentaire et Linguistique 1

Centre d'Etude et de Recherches en Informatique Linguistique ${ }^{2}$

\section{UNE GRAMMAIRE LOCALE DE L'EXPRESSION DES SENTIMENTS}

\section{Les expressions de sentiment}

Voici des phrases simples qui expriment des sentiments ${ }^{3}$ :

(1) Luc est anxieux

(2) Léa est en colère

(3) Luc a une drôle d'impression

Les phrases suivantes sont plus complexes :

(4) Les départs brusqués angoissent Luc

(5) Luc irrite Léa

(6) Les départs brusqués affectent Luc

elle diffèrent de (1)-(2)-(3) par la présence d'un agent ou d'une cause qui détermine le sentiment de Luc. L'agent ou la cause sont ici en position de sujet grammatical, ces termes sont en position d'objet direct dans :

(7) Luc (adore + déteste) les départs brusqués

La description que nous allons donner est une application directe de la théorie du lexique-grammaire dont le postulat fondamental localise les éléments de sens dans des phrases élémentaires et non pas dans les mots (M. Gross, 1975). Ainsi, la description de la formulation des sentiments consistera-t-elle en une grammaire locale, et non pas en un simple lexique des termes de sentiments. En conséquence, on considère qu'un lexique de noms de sentiments n'a pas d'autonomie, et donc que les noms doivent être entièrement intégrés aux familles de phrases présentées ici sous forme de grammaire. Ce point de vue a une traduction sémantique claire et quasi tautologique : un sentiment est toujours attaché à la personne qui l'éprouve. On peut formaliser cette association en la notant par un prédicat sémantique :

$\left(\mathbf{P}_{1}\right)$ Sent $(\mathbf{h})$

où le sentiment Sent est une fonction d'une variable $h$, qui correspond à des humains. Il existe alors autant de fonctions Sent que de sentiments. Une autre notation pour cette association est :

$\left(\mathbf{P}_{2}\right) \mathbf{P}($ sent, h)

où $P$ est une relation prédicative qui lie deux variables : un sentiment sent et un humain $h$.

1. UA 1576 du CNRS.

2. FIRTECH Industries de la langue, Universités Marne-la-Vallée et Paris 7.

3. Rappelons que l'effet dit performatif revient à signaler que ces phrases sont le fait d'un observateur qui rapporte sa perception qu'il a des sentiments de Luc. 
Il est clair que les deux notations sont équivalentes, si dans (2) nous fixons sent, alors P(sent, h) peut être noté $P_{\text {sene }}(h)$, fonction identique à $\left(P_{1}\right)$. On écrirait ainsi, pour $(1)$ :

Anxiété (Luc)

ou bien : P(anxiété, Luc) avec $\mathbf{P}_{\text {anxiéı́ }}($ Luc) = Anxiété(Luc)

Nous allons voir que les expressions de sentiment sont très nombreuses dans le lexique, et que leur étude quantitative précise soulève des problèmes empiriques et théoriques nouveaux ${ }^{4}$. Lors de la construction d'une grammaire locale comme celle des phrases exprimant des sentiments, la première difficulté est celle de l'énumération systématique des expressions, de telles phrases présentent en effet une variété considérable de formes. La source de variété la mieux identifiée est syntaxique au sens traditionnel. Si par exemple une phrase active de forme $N_{o} V N_{1}$, comme (4)-(5)-(6)-(7), sert à exprimer un sentiment, alors les formes grammaticalement associées comme les passifs, les interrogatives ou les relatives véhiculent la même signification :

(5) = Léa est irritée par Luc

= Par qui Léa est-elle irritée?

= la personne que Luc irrite, etc.

Ces descriptions relèvent de la représentation par lexique-grammaire.

Une seconde source de variation, bien que relevant aussi de la morpho-syntaxe, est plus compliquée à identifier. Un sentiment étant donné par un mot, ce mot peut être un verbe, un nom, un adjectif ou un adverbe :

\section{étonner, étonnement, étonnant, étonnamment}

Ces mots correspondent à un même sentiment, mais ils se construisent différemment : comment mettre en relation les constructions équivalentes où ils apparaissent ? On verra comment la notion de verbe support apporte une solution.

\section{Le lexique-grammaire}

Le lexique-grammaire des verbes du français (M. Gross, 1975 ; J.-P. Boons, A. Guillet, C. Leclère, 1976a, 1976b ; A. Guillet, C. Leclère, 1992) énumère les principales constructions des verbes du français. Il comporte des classes de verbes de sentiments ${ }^{5}$ (e.g. classes 4 et $12, c f$. figure 1, p. 72). Par exemple, on a recensé dans la table 4 de l'ordre de six cents verbes du type (4)-(5)-(6) et dans la table 12 une soixantaine du type (7). Mais une évaluation quantitative suppose la résolution de divers problèmes syntaxiques et la mise au point d'un système formel de représentation pour les variations lexico-syntaxiques habituellement considérées comme relevant de la morphologie dérivationnelle. Nous allons examiner ces questions.

L'étude de 12000 verbes courants ${ }^{6}$ du français a dégagé un certain nombre de traitss

4. Une étude lexicale a été faite par J. Picoche 1976, du point de vue diachronique. Notre étude est strictement synchronique.

5 . Ou verbes psychologiques : la difficulté de fixer de tels termes peut paraître seulement terminologique, il s'agit en fait d'une question sémantique profonde.

6. Notre notion informelle de « courant » réfère à plusieurs pratiques : d'une part la possibilité de porter sur une construction de verbes des jugements d'acceptabilité reproductibles, $d$ 'autre part une division du vocabulaire général (mots courants et autres) en trois niveaux de plausibilité d'usage (M. Garrigues, 1992). 


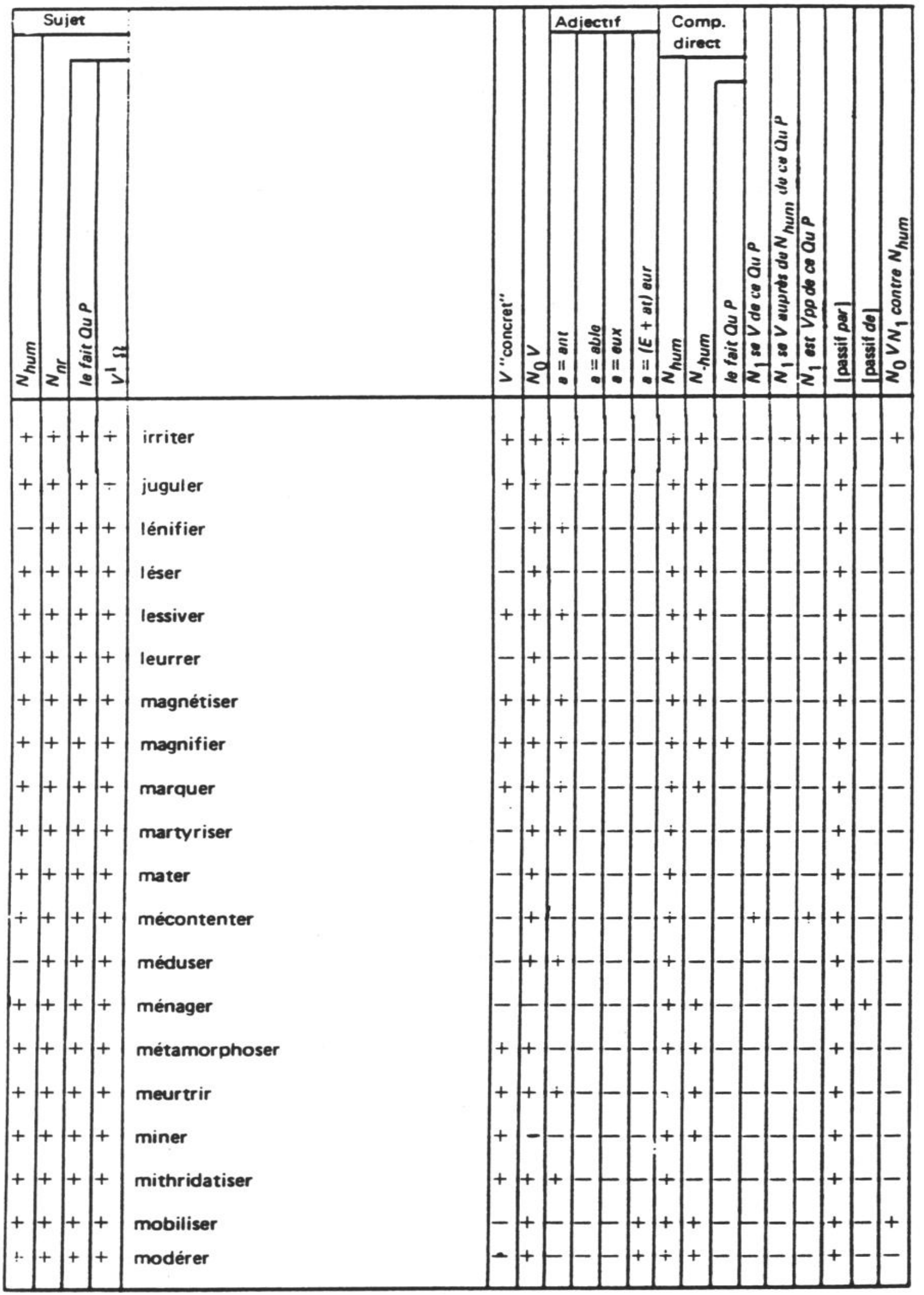

Figure 1

Table 4 : classe des verbes de sentiment 
tructuraux de leur construction. C'est ainsi que l'on a observé que la quasi-totalité des structures élémentaires du français étaient de l'une des formes :

$$
\begin{aligned}
& N_{o} V \quad \text { ou } N_{o} \text { être Adj } \\
& N_{o} V \text { Prép } N_{1} \quad \text { ou } N_{o} \text { être Adj Prép } N_{1} \\
& N_{o} V \text { Prép } N_{1} \text { Prép } N_{2}
\end{aligned}
$$

c'est-à-dire sujet-verbe, accompagné de zéro, un ou deux compléments essentiels ( objet »). Par ailleurs, les prépositions majoritaires sont * zéro *, à, de. Les phrases qui formulent des sentiments se conforment donc à ces schémas et une partie de la description consiste à classer les expressions selon les structures où elles entrent. Le problème de la classification se pose du fait que le terme de sentiment sent peut apparaître dans des positions syntaxiques nominales : $N_{o}, N_{1}, N_{2}$, verbales : $V$ ou adjectivales : $A d j$. De même, le terme humain, traduit par un groupe nominal, apparaîtra en position $N_{o}, N_{1}, N_{2}$. C'est ainsi que les deux exemples de tables que nous avons cités ont le type syntaxique commun $N_{o} V N_{1}$, avec comme terme de sentiment le verbe ; mais ils diffèrent par le terme humain qui est sujet $N_{o}$ dans la table 12, alors qu'il est complément direct $N_{1}$ dans la table 4.

\section{Les phrases à verbe support}

Les transformations qui modifient les parties du discours changent la nature grammaticale du terme de sentiment, et à un degré moindre celle du terme humain. Ce sont essentiellement les nominalisations de verbes et d'adjectifs et les adjectivations de verbes ${ }^{7}$. Exemples :

(1) Luc est anxieux = Luc a de l'anxiété (A. Meunier, 1977)

(2) Luc rage contre Léa = Luc est en rage contre Léa (D. de Négroni-Peyre, 1988)

Les verbes être (en) et avoir sont des verbes supports qui, au contraire des verbes distributionnels comme rager, ne peuvent pas correspondre au terme de sentiment. Pour notre classification, la distinction entre verbes distributionnels et verbes supports (M. Gross, 1981) est donc fondamentale. Dans les exemples suivants :

(3) Luc (éprouve + ressent) de l'anxiété

(4) L'anxiété (envahit + gagne + pénètre) Luc

les verbes sont supports, ils peuvent comporter des modalités de sens. On considère que (3) et (4) appartiennent au paradigme syntaxique (1) malgré quelques différences de sens, autrement dit on considère que les phrases (1), (3) et (4) partagent un invariant de sens que l'on a noté Anxiété(Luc). On remarquera que dans (1) et (3), $N_{o}$ est l'humain et $N_{1}$ est le sentiment, et que dans (4), l'ordre est inversé : $N_{o}$ est le sentiment et $N_{l}$ l'humain. Dans la forme équivalente :

(5) Il y a une certaine angoisse chez Luc

l'humain et le sentiment sont tous deux compléments. A ce point de la présentation, on notera bien que la seconde notation proposée, soit :

\section{P(Anxiété, Luc)}

met en évidence le rôle du verbe support : celui-ci matérialise le lien prédicatif $\mathbf{P}$.

7. Cette terminologie implique une orientation des transformations, or nos transformations ne sont pas orientées, ce sont des relations d'équivalence. Il résultera parfois de cette situation une difficulté terminologique pour le lecteur habitué à une orientation de ces processus. 
Les exemples (1) à (5) montrent bien la diversité syntaxique des phrases élémentaires exprimant les sentiments d'une personne ( $c f$. A. Balibar-Mrabti, 1995). Une partie de cette diversité est liée aux éléments lexicaux particuliers que sont les verbes supports. Il en existe en effet une certaine variété ne différant entre eux que par des modalités, clairement aspectuelles dans (4), mais le plus souvent stylistiques, comme c'est le cas pour les phrases :

$$
\begin{aligned}
& \text { Luc (éprouve + ressent) de la joie } \\
& \text { Luc }\left\{\begin{array}{l}
\text { rayonne } \\
\text { vibre } \\
\text { tremble }
\end{array}\right\} \text { de joie }
\end{aligned}
$$

Nous considérons de tels ensembles de phrases comme sémantiquement équivalents (cf. Y. Mathieu, 1995) et nous les représenterons de façon systématique par des graphes ou automates finis du type de ceux de la figure 2.

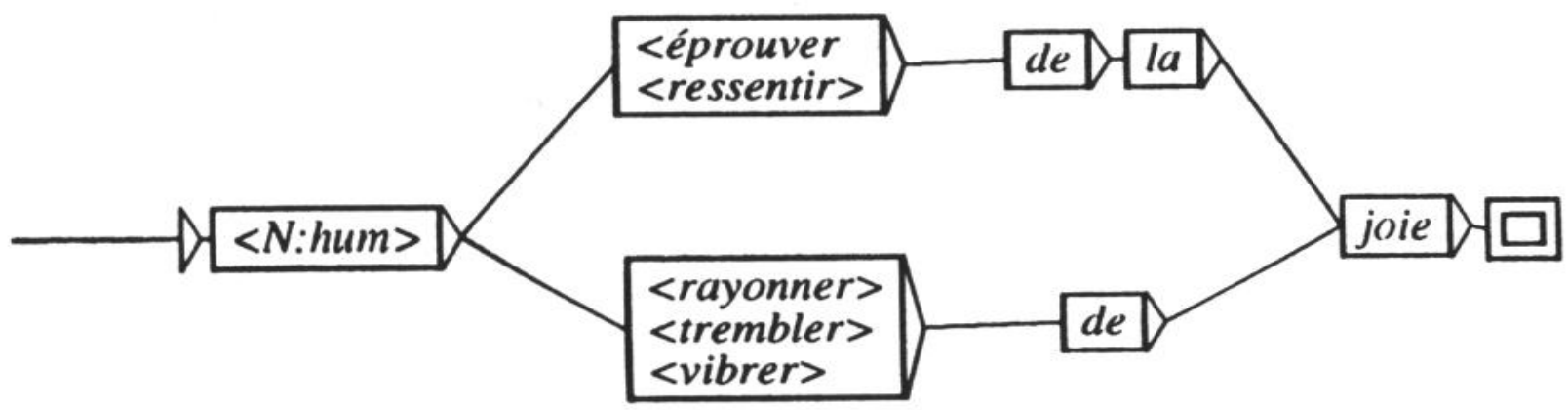

Figure 2

La figure 2 comporte des phrases du type de (4), donc « inversées " par rapport à celle de la figure 1.

Ces graphes se lisent de gauche à droite, de l'état initial à l'état final, chacun des parcours correspond à une phrase. Les parenthèses angulaire « < et " > signalent les parties devant faire l'objet d'ajustements grammaticaux (accords, contraction, élision). D'une part, ce mode de représentation permet de mettre en facteur les parties communes aux phrases équivalentes, ici l'humain et le sentiment, d'autre part ces graphes permettent d'introduire des restrictions détaillées sur la combinatoire des noms et des verbes en jeu. Nous allons en faire une utilisation systématique ${ }^{8}$. Mais cette combinatoire est extrêmement complexe, les restrictions de noms de sentiment à certains verbes sont nombreuses et tellement inattendues que dans un premier temps, nous les avions traitées comme des expressions figées, c'est-à-dire comme de simples listes. Dans la présente étude nous cherchons à mettre en valeur les propriétés syntaxiques communes à ces expressions et de ce fait, nous avons été amené à simplifier la représentation des restrictions lexicales. C'est ainsi que nous utilisons dans les graphes de grammaire l'approximation suivante : les sentiments sont classés selon les deux types : joie et rage, et nous donnons en annexe des listes de $N$ qui correspondent à ces classes. En fait, la classification des expressions de sentiment est avant tout un problème philosophique. On discute depuis bien longtemps de la séparation entre sentiments, sensations, impressions, qualités morales et autres états de l'âme ; on a également beaucoup spéculé sur la nature innée ou acquise des mécanismes que mettent en jeu toutes ces entités. Transposées au domaine des études littéraires, les mêmes questions conduisent à l'analyse d'expressions de sentiments observables dans les textes. L'étude que

8. Par ailleurs, ces graphes peuvent être immédiatement incorporés à un analyseur syntaxique qui les localisera automatiquement dans des textes (système INTEX, M. Silberztein, 1993). 
nous esquissons est duale du problème littéraire : nous recherchons dans le lexique et dans la grammaire du français les formes qui sont disponibles pour exprimer les sentiments et nous les décrivons systématiquement.

\section{Autres prédicats de sentiment}

Nous avons mentionné l'intervention d'un agent ou d'une cause dans des phrases exprimant des sentiments. Les prédicats sémantiques correspondants Caus sont donc plus complexes, on peut les noter de diverses façons, on écrira s pour le stimulus (cause ou agent), qui déclenche le sentiment sent chez une personne h. On pourra alors écrire :

- ou bien Caus(s, sent, h), prédicat à 3 variables,

- ou bien Caus(s, P(Sent, h)), prédicat à 2 variables dont l'une est elle-même un prédicat,

- et, si l'on fixe le sentiment comme en 1, d'autres notations sont encore possibles ${ }^{9}$.

Les phrases des tables 4 et 12 déjà signalées correspondent à ce prédicat, et on peut observer - d'autres formes syntaxiques de type causatif :

- des opérateurs causatifs classiques de la forme $N_{o} V(=$ La nouvelle fait $)$ s'appliquent à des phrases de type P(Sent, h) :

La nouvelle fait trembler Luc de joie

La nouvelle met de la joie en Luc

La nouvelle donne de l'angoisse à Luc

- d'autres opérateurs ont des effets syntaxiques encore différents, comme ceux des figures 3 et 4.

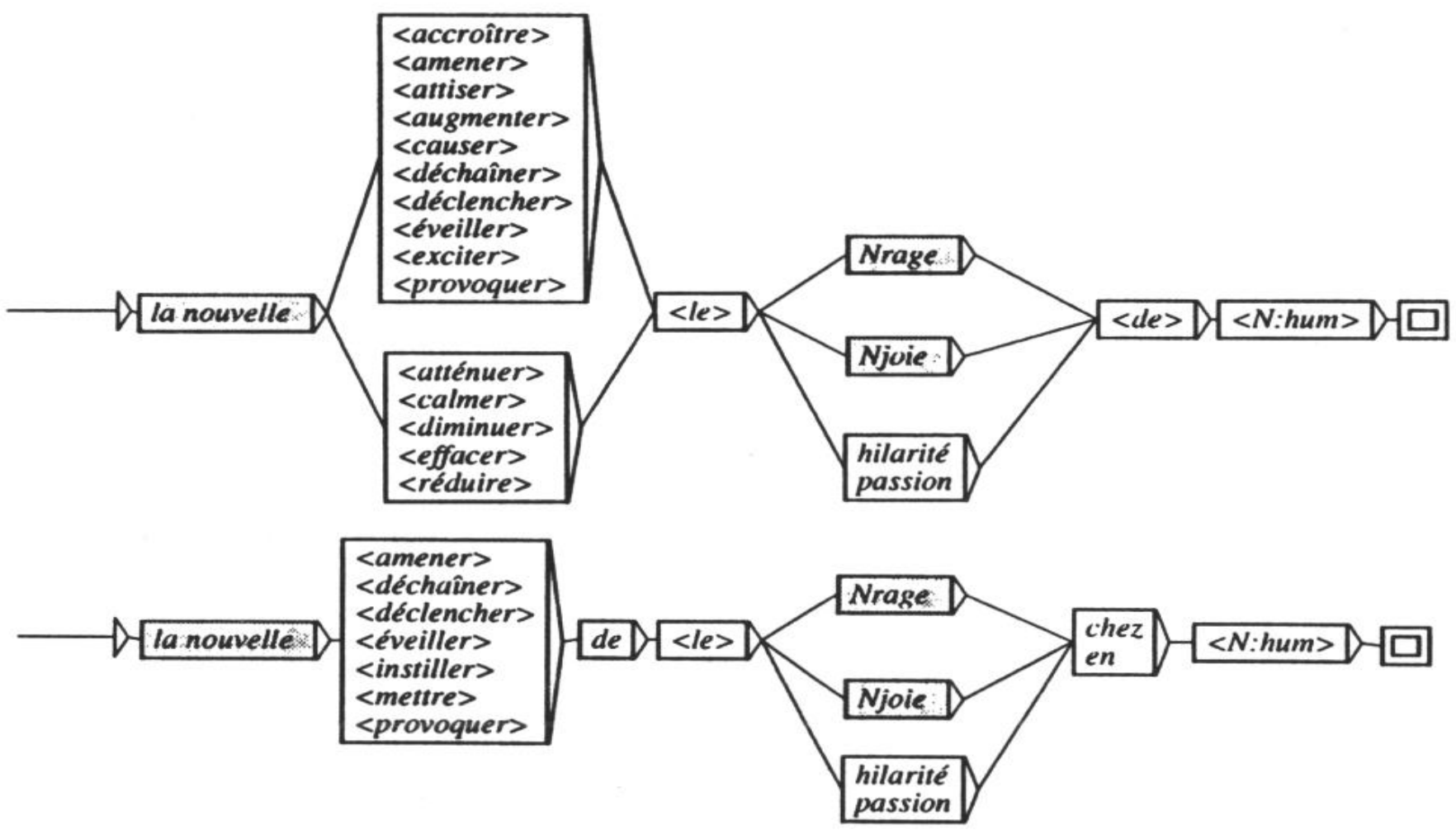

Figures 3, 4

9. Ces notations suggèrent la possibilité d'opérations de composition ou de calcul formel sur les prédicats et les variables. Pour qu'il en soit ainsi, diverses conditions doivent être remplies, et il n'est pas certain du tout que ce soit le cas ici. 
On a encore des exemples comme :

La nouvelle (inonde + remplit) Luc de joie

qui se présentent comme résultant de l'application d'un opérateur causatif à une phrase simple comme :

Luc est plein de joie

Les relations syntaxiques entre les phrases les plus simples et leurs causatives sont variées, comme on peut encore le voir sur l'exemple suivant :

(1) Léa (a + éprouve + ressent) de l'agacement

(2) Il y a de l'agacement (en + chez) Léa

(1) [Adjectiv.] = Léa est agacée

(1) $\left[N_{o}\right.$ causer $]=$ Cette situation a causé l'agacement de Léa

(2) $\left[N_{o}\right.$ causer $]=$ Cette situation a causé de l'agacement (en + chez) Léa

De telles phrases peuvent être sujet d'un verbe support d'occurrence (Z.S. Harris, 1976, M. Gross, 1990) introduisant un complément ou adverbe de cause :

\section{Que Léa ait de l'agacement se produit devant cette situation}

d'où dériveront les phrases :

Léa a de l'agacement devant cette situation.

[Adjectiv.] = Léa est agacée devant cette situation

Nous appelons devant cette situation un complément de cause par analogie avec le complément d'agent du Passif, en effet nous retrouvons dans ces phrases le sujet causatif de l'opérateur $\left[N_{o}\right.$ causer], permuté et précédé de dans. Cette analyse est justifiée par l'interdiction du même complément dans les formes causatives suivantes :

* Ce texte agace Léa devant la situation

* Ce texte a causé l'agacement de Léa devant cette situation ${ }^{10}$

Par ailleurs nous avons signalé l'effet performatif et donc indiqué que les phrases étaient émises par un locuteur qui n'est pas forcément lié à la personne affectée par le sentiment. Cette situation est explicite avec certains verbes comme :

Léa a perçu la joie de Luc

= Léa a perçu de la joie en Luc

ainsi que ceux de la figure 5 (cf. p. 77).

D'une manière générale, les phrases spécifiques d'expression de sentiment peuvent se combiner avec des phrases quelconques ", conduisant à des variétés nouvelles de phrases. Ainsi, des phrases comme :

Léa a fait part à Guy de la joie de Luc

La joie de Luc agace Léa

comportent le prédicat Joie(Luc). L'introduction de tels prédicats dans les positions syntaxiques $N_{o}, N_{1}, N_{2}$ posent des problèmes variés (M. Mohri, 1992). Pour ces deux exemples, on a recours à des dérivations ayant pour point de départ une complétive :

Léa a fait part à Guy de ce que Luc avait une certaine joie

Que Luc ait une certaine joie agace Léa

10. Le même groupe nominal en agacement est autorisé dans d'autres contextes : L'agacement de Léa devant cette situation étonne Luc.

11. Aux contraintes distributionnelles près. 


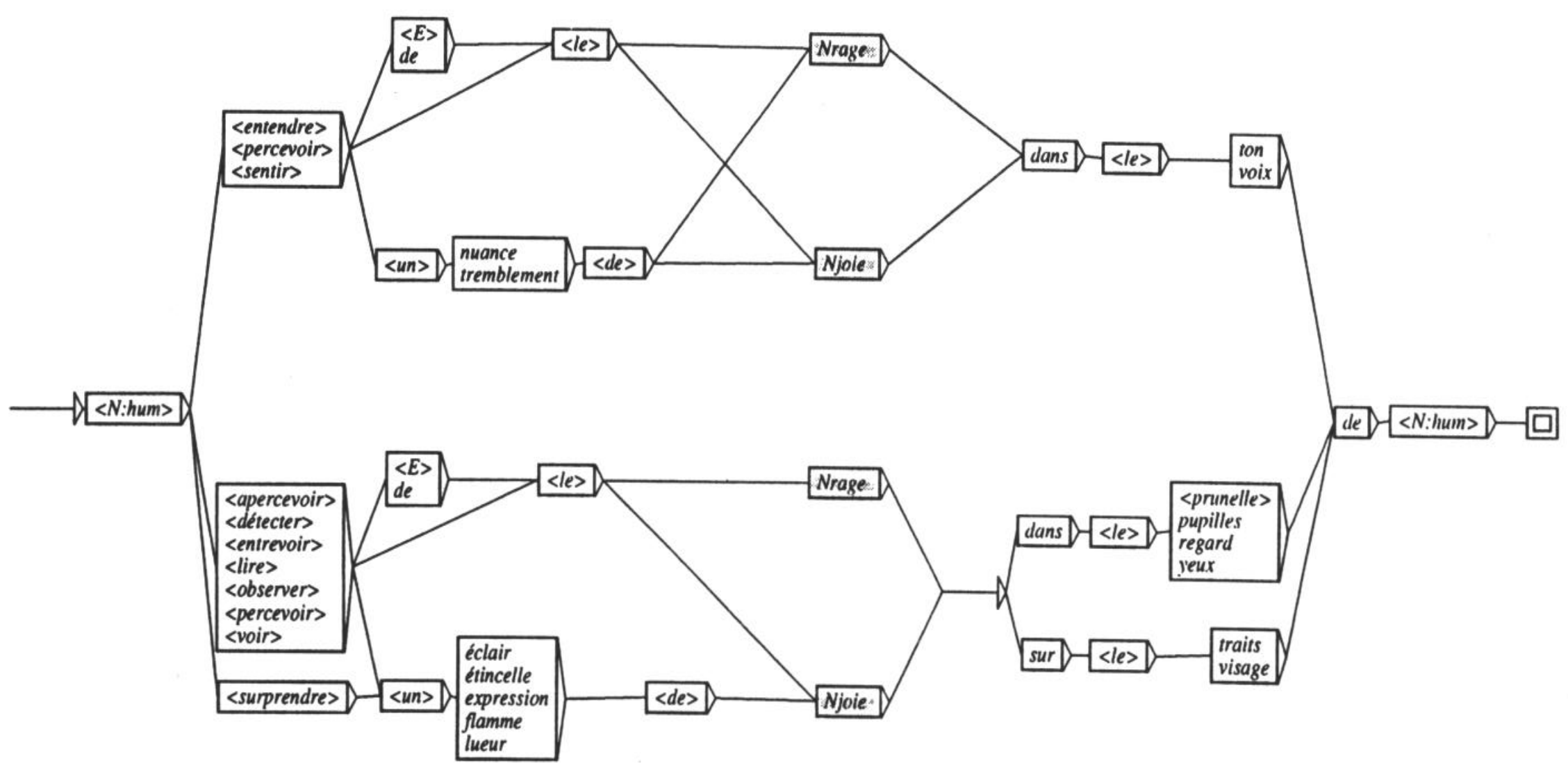

Figure 5 
La complétive qui contient la phrase de sentiment à verbe support subit alors les transformations :

$\begin{array}{lll} & & \text { (ce) que Luc a une certaine joie } \\ {[\text { Relativation }]} & = & \text { la joie que Luc a } \\ {\left[N_{\text {o }}\right]} & = & \text { la joie qu'a Luc } \\ {[\text { Vsup Réd. }]} & = & \text { la joie de Luc }\end{array}$

Mais cette dérivation ne s'appliquera pas à d'autres phrases comme par exemple :

Léa a décrit à Guy la joie (qu'a + de) Luc

\section{Formes stylistiquement enrichies}

Une nouvelle variété de formes est due à la présence de substantifs de divers types qui peuvent apparaître dans la position formelle d'un déterminant de $N$ sent et $N$ hum : pour les Nsent ce sont des $N$ dét comme éclair (de rage), nuance (de reproche) ou des classifieurs comme sentiment, sensation, expression, et pour les Nhum ce sont surtout des noms de partie du corps $N p c($ yeux $)$ ou autres inaliénables. Ils sont insérés dans les formes de base dont la structure globale, donnée en 2, n'est pas modifiée ; on a par exemple :

$N_{o} V N_{1}=:(\text { Un éclair de rage })_{o}$ traversa (le regard de Luc)

ou bien avec un sujet $N_{o}$ causatif :

(1) $\quad N_{o} V(L E N p c \text { de Nhum })_{1}$ de (Dét Ndét de Nsent) $)_{2}=$ :

(1a) La nouvelle illumina le visage de Luc d'une expression de joie

Les formes suivantes sont un peu moins naturelles :

(2) $\quad N_{o} V(\text { Dét Ndét de Nsent), Loc(LE Npc de Nhum })_{2}=$ :

(2a) La nouvelle mit une expression de joie dans le regard de Luc

?La nouvelle (étala + répandit) une expression de joie sur le visage de Luc ${ }^{12}$

De telles structures sont parmi les plus redondantes dans la mesure où les déterminations Ndét n'apportent guère d'information. Différentes opérations réduisent la redondance et conduisent aux formes courtes. Ces opérations sont :

- des effacements de constante : [Ndét z.], [Npc z],

- des transformations par lesquelles un terme, en général l'agent (ou le causatif) peut disparaître.

Comme pour toutes les transformations, ces opérations présentent des restrictions lexicales, mais ces contraintes ne remettent pas en cause la généralité des mécanismes. Par exemple, l'application à $(2 \mathrm{a})$ des deux effacements ${ }^{13}$ de $N p c$ et $N$ dét conduit à :

La nouvelle mit de la joie en Luc

? La nouvelle illumina Luc de joie.

De même on a :

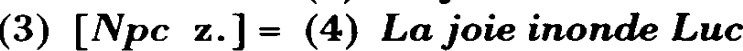

(5) Une rage soudaine remplit (le cour + le regard + les yeux) de Luc

(5) $[$ Npc z. $]=(6)$ Une rage soudaine remplit Luc

12. On remarquera que le passé simple, d'emploi limité aujourd'hui, est naturel dans ces clichés.

13. Ầ quelques ajustements morphophonémiques près. 
Dans ce cas, le $N p c$ qui est effacé est déterminé à l'équivalence près :

cour $=$ regard $=$ yeux

et cette équivalence lexicale ${ }^{14}$ est caractéristique des variantes observées dans les expressions figées.

Considérons encore les formes prépositionnelles :

(Dét Ndét de Nsent) ${ }_{o}$ V Prép (Dét Ndét de Nhum) $=$ :

Une (expression + lueur) de joie passa dans le regard de Luc

Un frisson de rage (courut + passa) $(E+$ tout $)(E+\grave{a})$ le long de la colonne vertébrale de Luc

dans ces exemples, les $N$ dét ne sont pas effaçables :

?*(La joie + Une joie profonde) passa dans le regard de Luc

*Un frisson de rage (courut + passa) $(E+\grave{a})$ Luc

Toutefois, il existe des formes partiellement réduites :

Une expression de (béatitude + bonheur) s'étalait sur (le visage + les traits) de Luc

$=$ (La béatitude + le bonheur) s'étalait sur (le visage + les traits) de Luc

*Une expression de béatitude s'étalait sur Luc

et d'autres compléments peuvent être réduits :

L'expression d'une joie intense émanait de (le visage + le regard + les yeux) de Luc

= Une joie intense émanait de Luc

Les $N$ dét introduisent des restrictions sur les combinaisons avec les termes de sentiment, on perçoit ainsi des différences de naturel entre des formes identiques :

Une lueur de satisfaction brilla dans le regard de Luc

? Une lueur de (bonheur + cruauté + dédain) brilla dans les yeux de Luc

La délimitation des combinaisons de $N$ sent avec le verbe et le déterminant $N$ dét se présente donc comme un problème classique de détermination de distribution, toutefois limité à un ensemble fermé de termes. Aussi les irrégularités constatées et la complexité des données justifient-elles une représentation par grammaires locales plutôt que par tables syntaxiques. Les graphes des figures 5-6-7 comportent les restrictions que nous venons de discuter.

Notons que par ailleurs, il existe des formes figées qui constituent des expressions de sentiments mais qui n'en utilisent pas le vocabulaire, elles sont en général isolées et ne peuvent donc être décrites que par des listes :

Luc prend son pied

Les départs brusqués (soulèvent + touchent) le cour de Luc

Les départs brusqués lui coupent la chique

\section{Les transformations}

\section{LES RESTRUCTURATIONS}

On observe :

- la restructuration de sujet :

La colère de Luc (augmente + disparâ̂t + éclate)

= La colère (augmente + disparâ̂t + éclate) en Luc

14. D'une manière générale, il semble nécessaire de distinguer des sentiments extériorisés et des sentiments purement intérieurs. L'introduction de cette distinction pourrait séparer cœur de regard = yeux. 
- la restructuration de complément :

La nouvelle (déclenche + éveille) de la joie en Luc

= La nouvelle (déclenche + éveille) la joie de Luc ${ }^{15}$

Les figures 3 et 4 donnent d'autres exemples de ces paires.

\section{La Conversion [Conv]}

La relation formelle de Conversion (C. Fillmore, 1968) ou relation Standard-Croisé a été étudiée pour le français par J.-P. Boons, A. Guillet, C. Leclère 1976a (Table 34LO), elle s'observe sur des phrases comme :

\section{Les fourmis grouillent dans l'allée}

[Conv $\left.N_{o} N_{1}\right]$ = L'allée grouille de fourmis

Luc charge des caisses dans le camion

[ Conv $N_{1} N_{2}$ ] = Luc charge le camion de caisses

Les phrases suivantes :

(1) La joie brille dans les yeux de Luc

[Conv $N_{o} N_{1}$ ] = (2) Les yeux de Luc brillent de joie

ont été décrites comme ay ant des sujets figés et à ce titre figurent dans une table CO, mais elles y posent des problèmes de représentation. D'une part, le caractère figé de ces expressions n'est pas clair :

- la position de yeux semble figée : ainsi il n'est pas possible de substituer à yeux : deux yeux ou l'oil droit, comme ce serait le cas par exemple avec le verbe irriter, on comparera :

\section{*La joie brille dans (les deux yeux + l'oeil droit de Luc) \\ Les deux yeux de Luc sont irrités \\ L'œil droit de Luc est irrité}

Cependant, on acceptera dans la position figée du groupe le regard, d'autres groupes comme les pupilles et les yeux clairs, avec un effet stylistique :

La joie brille dans le regard de Luc

? La joie brille dans les (yeux clairs + pupilles) de Luc

- la position du nom de sentiment $N$ sent apparaît elle aussi comme susceptible de variations lexicales, on accepte :

LE Nsent brille dans (les yeux + le regard) de Luc

[Conv $\left.N_{o} N_{l}\right]=($ Les yeux + Le regard $)$ de Luc brille(nt) de Nsent

avec des noms de sentiments variés (aussi bien Njoie que Nrage) :

Nsent $=:$ bonheur + désir + envie + enthousiasme + excitation + joie + colère + rage + haine + ressentiment + convoitise + satisfaction + impatience

mais il faut signaler des restrictions difficilement prévisibles du point de vue du sens :

$?^{*}$ La (peine + souffrance + tristesse) brille dans les yeux de Luc

15. Ces différences de formes pourraient s'expliquer par les différences de Vsup sous-jacents (cf. exemples (1)-(2) en 3 . 
De plus, les deux prédéterminants un éclair de, une lueur de peuvent précéder les Nsent acceptés :

(Un éclair + une lueur) de joie brille dans le regard de Luc

Un élément de complexité supplémentaire provient du fait que le verbe briller présente des variantes : étinceler, luire ${ }^{16}$ dans la relation :

(1a) La joie (étincelait + luisait + rayonnait) dans le regard de Luc

(la) [Conv $\left.N_{o} N_{1}\right]=(2 a)$ Le regard de Luc (étincelait + luisait + rayonnait) de joie Le $N p c=$ : visage est autorisé, mais pas avec briller :

*La joie brillait (dans + sur) le visage de Luc

On a par ailleurs des variations avec des Prép de type locatif :

La joie rayonnait sur le visage de Luc

La combinaison $V$ Prép possède également la variante directe :

(3) La joie (éclairait + illuminait) (le regard + les yeux) de Luc

et cette construction directe $n$ 'a pas de forme converse. On observe cependant un changement de forme du $V$ qui se présente comme une forme passive en se (M. Gross, 1968) :

(3a) (Le regard + Le visage) de Luc s'illumina de joie

mais on analysera ces phrases différemment. On considèrera la forme longue, à sujet causatif :

(4) $N_{o} V N_{1}$ de $N_{2}=$

La nouvelle illumina de joie (le regard + le visage) de Luc

et on y appliquera la transformation [se-moyen], ce qui donne :

(4) $\left[\right.$ se-moyen] $=N_{1}$ se $V$ de $N_{2}=:(3 a)$

\section{LA TRANSFORMATION INSTRUMENTALE [Inst]}

Il existe une transformation qui place certains compléments instrumentaux $N_{2}$ en position sujet :

Luc recouvre la tache de sable noir

[Inst ] = Du sable noir recouvre la tache

La forme (3) s'obtient par cette transformation. Appliquée à (4) on obtient :

(4) $\left[\right.$ Inst] $=N_{2} V N_{1}=:$ (3)

Il existe d'autres phrases de la forme (4) :

(5) Un sourire illuminait de joie le (regard + visage) de Luc

Luc est le sujet de sourire dans (5), et il est clair que dans (4) et (5) les sujets ne sont pas de même nature. On aurait de même :

(6) = La nouvelle éclaira les yeux de Max d'une lueur de joie

(6) $[$ se-moyen] = Les yeux de Max s'éclairèrent d'une lueur de joie

16. Luire est moins naturel, encore moins avec le $N$ dét lueur. 
(6)[Inst] = Une lueur de joie éclaira les yeux de Max

Les règles et contraintes que nous venons d'examiner sur quelques exemples sont d'une grande généralité, elles interviennent entre autres dans les phrases des figures 6 et 7 (p. 83-84). Nous en rencontrerons quelques autres d'application plus restreintes.

Signalons encore deux transformations importantes :

L'ADJECTIVATION DE VERBES (E. Laporte, 1992).

(2) Les yeux de Luc brillent de joie

(2) $[\mathrm{V}=\mathrm{A}]=$ Les yeux de Luc sont brillants de joie

mais la phrase (1) dont (2) est la converse n'a pas de forme adjectivée :

(1) $[\mathrm{V}=\mathrm{A}]=*$ La joie est brillante dans les yeux de Luc

LES NOMINALISATIONS du type :

La joie (luit + pétille) dans les yeux de Luc

(7) $[\mathrm{V}=\mathrm{N}]=$ Il y a (une lueur + un pétillement) de joie dans les yeux de Luc

qui déplacent le verbe en position de déterminant. On notera que ces formes nominales peuvent être autonomes (i.e. sans verbes associés) comme :

Il y a un éclair de joie dans les yeux de Luc

Nous résumons l'effet de diverses opérations que nous venons de discuter en donnant un exemple de dérivation plus complète. Nous partons de :

(8)

La nouvelle remplit le cour de Luc de rage

(8)[Passif] = (9) Le cour de Luc est rempli de rage (E + par ton geste)

(8) [se moyen] = (10) Le cour de Luc se remplit de rage

(8)[Instr.] = (11) La rage remplit le cour de Luc

(9) $[\mathrm{VPp}=\mathrm{A}]=$ (12) Le cour de Luc est plein de rage

La règle d'effacement [Det $N$ dét de z.] est applicable aux phrases (8) à (11). Toutefois, les phrases :

(10z) Luc se remplit de rage

(11z) La rage remplit Luc

sont moins naturelles que d'autres comme :

(8z) La nouvelle remplit Luc de rage

(12z) Luc est plein de rage

\section{Une contrainte interne aux expressions de sentiment}

Précisons la structure de certaines des formes rencontrées. Reprenons la phrase :

(1) La joie brille dans les yeux de Luc

et examinons certaines variations de déterminants, plus particulièrement les adjectifs possessifs. On accepte :

(2) La joie brille dans ses yeux 


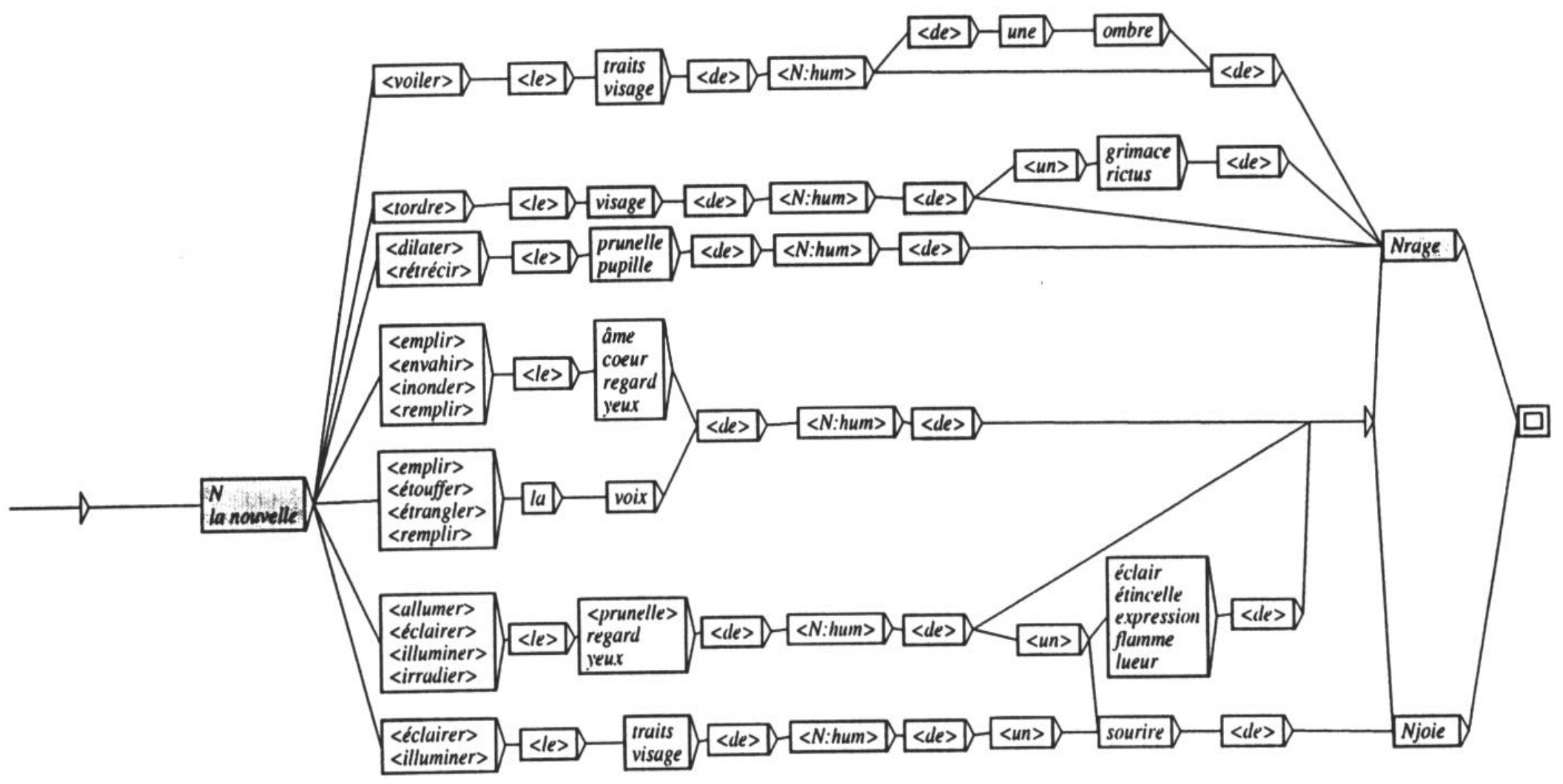

Figure 6 


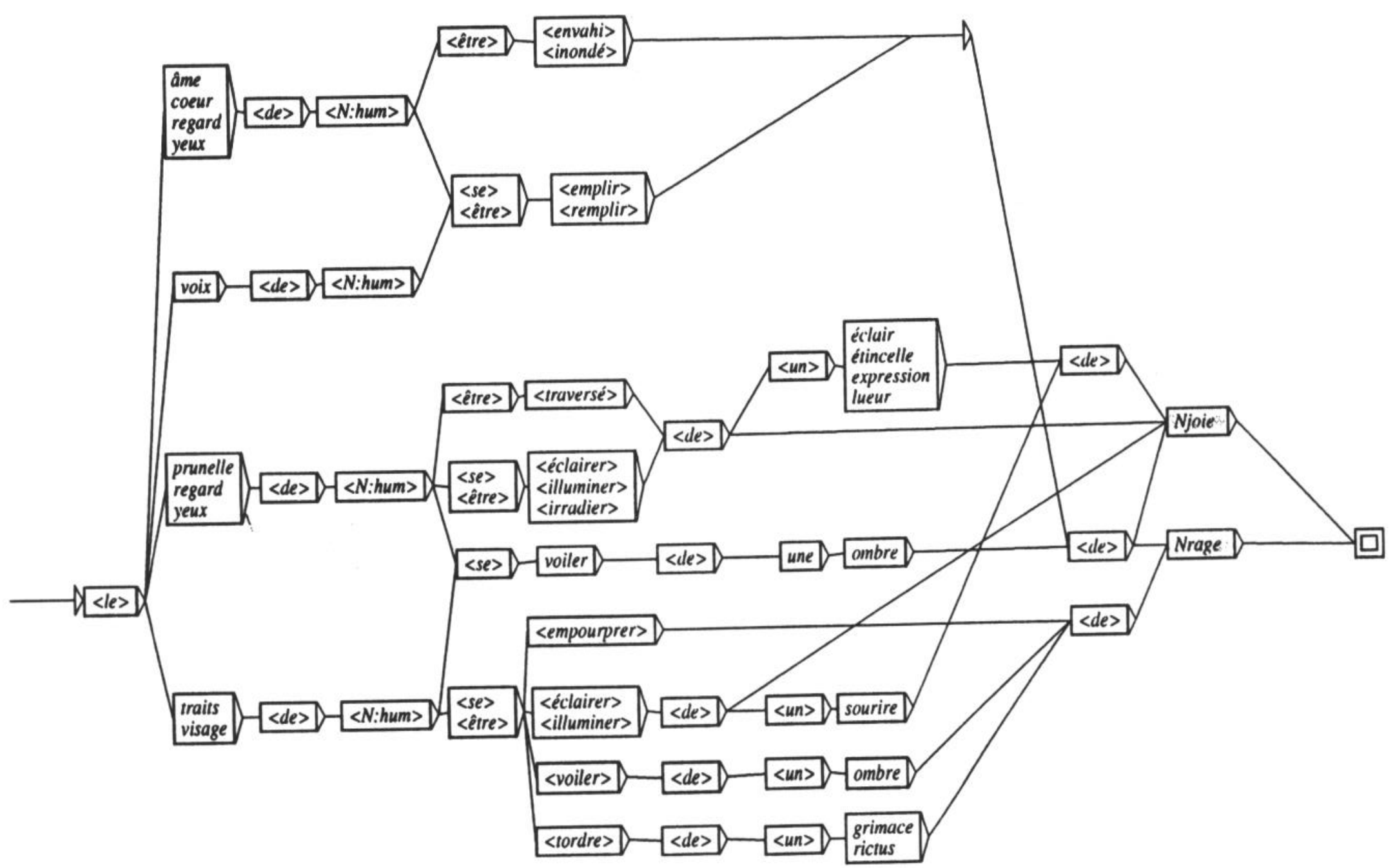

Figure ? 
obtenue de (1) par la règle générale de formation de Poss :

$N$ de LUI = Poss $N$

On accepte aussi :

(3) La joie de Luc brille dans ses yeux

(4) Sa joie brille dans ses yeux

On a $(3)=(4)$ par la même règle de formation de Poss. La particularité de ces structures est la coréférence obligatoire entre Luc et Poss, quelle que soit la position de Luc :

(3a) * La joie de Luc brille dans tes yeux

(4a) * Ta joie brille dans les yeux de Luc

Aussi, nous sommes amené à considérer la structure de base :

(B) La joie de Luc brille dans les yeux de Luc

où les deux occurrences de $L u c$ ont même référent. De cette forme sont dérivées les formes (1), (3) et (4) par application de la règle de formation de Poss. La forme (2) s'obtient de (B) par une autre règle :

$N$ de LUI $=N \quad$ avec $\quad N=: N$ sent $=:$ joie

règle qui ne s'applique pas ici pour $N=: N p c=:$ yeux. On notera toutefois l'interdiction de coréférence " en avant ":

*Sa joie brille dans les yeux de Luc (sa = de Luc)

Dans la position du nom yeux (regard, visage), ce sont les déterminants indéfinis qui sont acceptés, ils sont alors génériques :

(5) La joie brille toujours dans (un + un tel + des) regard(s) (E+d'enfant)

(6) La joie brille dans les regards (E+des gens)

Le complément de nom, indéfini, est alors facile à omettre. Dans la position du nom joie, le déterminant indéfini un certain est obligatoire, autrement dit un modifieur est obligatoire en présence de un :

(7) Une (certaine joie + joie intense) brillait dans son regard

*Une joie brillait dans le regard de Luc

La forme à sujet indéfini permettrait d'analyser le complément de nom de joie par relativation, même si la forme intermédiaire n'est pas naturelle :

\section{? La joie que (avait Luc + il avait) brillait dans son regard}

L'introduction de la proposition relative (ou du complément de nom) ne résout pas tous les problèmes d'interprétation de la phrase (1); en effet dans la phrase (7), il est clair que la joie est celle de Luc. Il nous est difficile de pousser plus loin la décomposition de la phrase (7), c'est-à-dire selon la forme minimale :

(C) Une certaine joie brillait dans un certain regard

Cette forme n'est pas acceptée et la cause en est claire : ni joie ni regard ne sont des substantifs autonomes. Il nous faut donc faire l'hypothèse que $(\mathrm{C})$ est une séquence support, c'est-à-dire l'équivalent de verbes supports simples comme avoir ou Il y avoir dans :

$N_{o}$ a une certaine joie

Il y a une certaine joie en $N_{2}$

Ces observations syntaxiques sur la coréférence, c'est-à-dire sur un lien obligatoire entre un nom de sentiment et un humain qui l'éprouve viennent donc encore à l'appui de notre hypothèse de départ qui interdisait la dissociation entre ces deux termes et ainsi oblige à prendre pour point de départ des analyses les phrases élémentaires. Ces faits confirment 
indirectement le principe fondamental du lexique-grammaire : le sens minimal n'est pas localisé dans les mots mais dans des phrases ${ }^{17}$.

\section{REFERENCES}

Balibar-Mrabti, Antoinette, 1995 : Langue française, ce numéro, Paris : Larousse.

Boons, Jean-Paul, GUILLET, Alain, LECLERE, Christian, 1976a : La structure des phrases simples en français. I. Constructions intransitives, Genève : Droz, 377 p.

Boons, Jean-Paul, GuILlET, Alain, LECLÈRE, Christian, 1976b : La structure des phrases simples en français. III. Constructions transitives, Paris : Rapport de recherches du LADL, ${ }^{\circ} 6,85$ p., tables et index, $58 \mathrm{p}$.

Fildmore, Charles J., 1968 : The Case for Case, Universals in Linguistic Theory, E. Bach \& R. Harms eds., Georgetown : Georgetown University Press.

GaRrigues, Mylène, 1992 : Dictionnaires hiérarchiques du français, Langue française $\mathbf{n}^{\circ}$ 96, Paris : Larousse, pp. 88-100.

GIRY-SCHNEIDER, Jacqueline, 1978 : Les nominalisations en français. L'opérateur FAIRE dans le lexique, Genève : Droz, 353 p., tables : $61 \mathrm{p}$.

GIRY-SCHNEIDER, Jacqueline, 1987 : Etude de prédicats nominaux en français. Les constructions faire $N$, Genève : Droz, 399 p.

Gross, Maurice, 1968 : Grammaire transformationnelle du français. 1-Syntaxe du verbe, Paris : Larousse, réimpression Cantilène-Distique, $183 \mathrm{p}$.

Gross, Maurice, 1975 : Méthodes en syntaxe, Paris : Hermann, 412 p.

Gross, Maurice, 1981 : Les bases empiriques de la notion de prédicat sémantique, Formes syntaxiques et prédicats sémantiques, A. Guillet et C. Leclère eds., Langages, $n^{\circ} 63$, Paris : Larousse, pp. 7-52.

Gross, Maurice, 1982 : Une classification des phrases figées du français, Revue québécoise de linguistique, vol. 11, $\mathbf{n}^{\circ}$ 2, Montréal : Presses de l'Université du Québec à Montréal, pp. 151185.

Gross, Maurice, 1990 : Grammaire transformationnelle du français. 3-Syntaxe de l'adverbe, Paris : ASSTRIL, 670 p.

Guillet, Alain, LeClêRe, Christian, 1992 : La structure des phrases simples en français. II Constructions transitives locatives, Genève : Droz, 445 p.

Harris, Zellig S., 1976 : Notes du cours de syntaxe, Paris : Le Seuil, 237 p.

LAPORTE, Eric, 1992 : Adjectif en -ant dérivés de verbes, Langue française $n^{\circ}$ 96, Paris : Larousse, pp. 30-43.

Mathieu, Yannick, 1995 : Langue française, ce numéro, Paris : Larousse.

MOHRI, Mehryar, 1993 : Analyse et représentation par automates de structures syntaxiques composées, Thèse de doctorat, LADL et CERIL, Université Paris 7 : Paris, 366 p.

Meunier, Annie, 1977 : Sur les bases syntaxiques de la morphologie dérivationnelle, Lingvisticae Investigationes, vol. I, $\mathrm{n}^{\circ}$ 2, Amsterdam : J. Benjamins B.V., pp. 287-332.

NGGroni-Peyre de, Dominique, 1978 : Nominalisation par être en et réflexivation (admiration, opposition, révolte et rage), Lingvisticae Investigationes, vol. II, $\mathbf{n}^{\circ} 1$, Amsterdam : J. Benjamins B.V., pp. 127-164.

17. Ce qui n'exclut pas la possibilité de phrases comme :

Luc discute de la (joie + rage)

où syntaxiquement, joie et rage apparaissent comme des objets autonomes. En fait, ces emplois génériques peuvent s'analyser à partir d'éléments phrastiques comme ceux de :

Luc discute de la (joie + rage) qu'ont les humains 
PICOChe, Jacqueline, 1976 : Le vocabulaire psychologique dans les Chroniques de Froissart, Paris : Klincksieck, 238 p.

SILBERZTEIN, Max, 1993 : Dictionnaires électroniques et analyse automatique de textes, Le système INTEX, Paris : Masson, 233 p.

\section{ANNEXE}

Noms de type joie :

allégresse, béatitude, bonheur, compassion, compréhension, complicité, confiance, confort, connivence, contentement, espérance, espoir, étonnement, excitation, fierté, gaité, gratitude, intelligence, joie, jouissance, malice, orgueil, pitié, plaisir, ravissement, réconfort, satisfaction, sérénité, surprise.

Noms de type rage :

abattement, amertume, angoisse, anxiété, apathie, chagrin, colère, convoitise, déception, découragement, dédain, dégoût, dépit, désespoir, détresse, désir, douleur, ennui, envie, épouvante, fatigue, frayeur, frustration, fureur, gêne, haine, honte, horreur, impatience, impuissance, inconfort, indignation, injustice, inquiétude, jalousie, lassitude, malaise, mécontentement, mélancolie, mépris, nervosité, panique, peur, rage, regret, rejet, remord, révolte, sinistrose, terreur, tristesse, trouille. 